\title{
SEMIREGULAR VARIABLES IN THE GALACTIC BULGE
}

\author{
M. SCHULTHEIS ${ }^{1}$, Y. K. NG ${ }^{2}$, J. HRON ${ }^{1}$, H. HABING ${ }^{2}$ \\ ${ }^{1}$ Institut für Astronomie, Wien, Austria \\ $\left({ }^{2}\right)$ Leiden Observatory, the Netherlands
}

\section{Observations ${ }^{1}$}

Near Infrared photometry (JHKL'M) of 78 SRVs in field \#3 (PG3) of the Palomar Groningen Variable Star Survey $\left(1=0^{\circ}, \mathrm{b}=-10^{\circ}\right)$ was obtained at the ESO 1-m telescope, La Silla, Chile.

\section{Results}

The Bulge SRVs were compared with the Bulge Miras (Blommaert 1992) and the field SRVs (Kerschbaum\&Hron 1994). In the $(\mathrm{H}-\mathrm{K}) /(\mathrm{J}-\mathrm{H})$ plane one can see some difference in the colour between the Bulge Miras and the Bulge SRVs. Bulge Miras are slightly redder in $\mathrm{H}-\mathrm{K}$ and bluer in $\mathrm{J}-\mathrm{H}$ than Bulge SRVs. The Bulge SRVs cover a wide range in this two colour diagram but are concentrated mainly in the same region as field SRVs. The position of $\mathrm{M}$ giants in a $(\mathrm{J}-\mathrm{H}) /(\mathrm{H}-\mathrm{K})$ plot is sensitive to surface gravity, atmospheric extension and chemical abundance. Metal-richer stars get a shift in the $(\mathrm{J}-\mathrm{H}) /(\mathrm{H}-\mathrm{K})$ plane towards bluer $\mathrm{J}-\mathrm{H}$ and redder $\mathrm{H}-\mathrm{K}$. By photometric means there is no metallicity difference between Bulge stars and field stars visible.

In the $(\mathrm{J}-\mathrm{H}) /(\mathrm{K}-\mathrm{L})$ plane the field SRVs are intermediate between the field SRVs and the Bulge SRVs. There is a clear separation between the three groups in $\mathrm{K}-\mathrm{L}$ as well as in $\mathrm{J}-\mathrm{H}$. This is caused partly by a temperature difference as well as a different atmospheric extension (Bessel et al. 1989). The separation in K-L could be enhanced by the different thickness of the circumstellar shell as with longer periods (Miras) the mass loss increases.

\footnotetext{
${ }^{1}$ supported by ASF project P9638-AST
} 


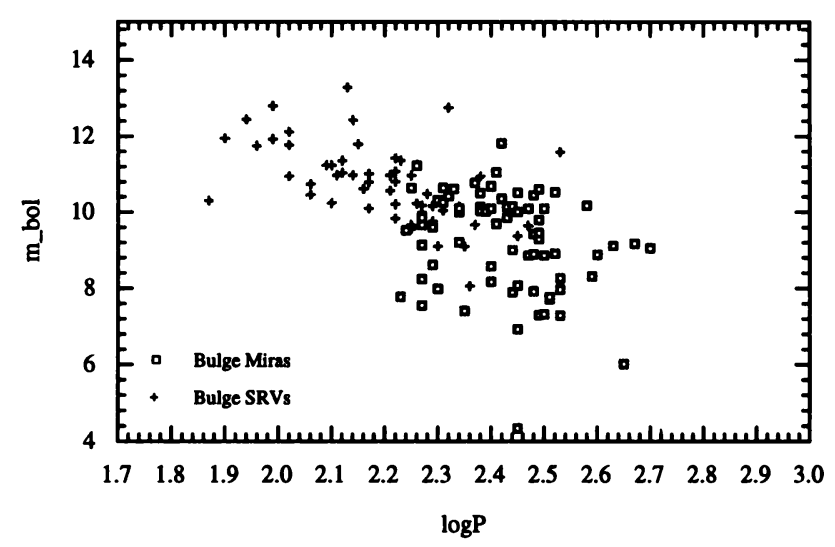

Figure 1. $\mathrm{m}_{\mathrm{bol}}$ is plotted against $\log \mathrm{P}$. The apparent bolometric magnitudes were derived by fitting a blackbody curve through JHKLM. The large scatter for the Bulge Miras can be explained by contamination of foreground stars. By excluding them, Bulge Miras follow the same PL relation as Bulge SRVs

Bulge SRVs and Bulge Miras obey the same $\mathrm{P} /(\mathrm{J}-\mathrm{K})$ relation, which is slightly above the $\mathrm{P} /(\mathrm{J}-\mathrm{K})$ relation for Miras in the LMC (Feast 1989). For longer periods the stars are cleary off the relation, because the dust shell becomes dominant. Field SRVs are redder in J-K than Bulge SRVs. In contrast to the Bulge SRVs, the slope of the PC relation for field SRVs is flatter. The same behaviour shows up in a $(\mathrm{J}-\mathrm{H}) / \log \mathrm{P}$ diagram where the separation of the three groups is even much clearer.

Bulge Miras as well as Bulge SRVs follow a period-luminosity relation (Fig. 1). This stresses the suggestion that Bulge SRVS are an extension of the Bulge Miras towards shorter periods. The scatter of the Bulge Miras is much higher than for the Bulge SRVs. This is caused by contamination of foreground stars and also by the much higher uncertainties in the determination of $\mathrm{m}_{\mathrm{bol}}$. Miras have larger amplitudes than SRVs and the scatter in the bolometric magnitude can reach nearly $1^{\mathrm{m}}$.

For all SRVs in PG3 low resolution spectra were obtained. Besides a spectral classification of these stars we are going to obtain radial velocities for these stars in order to compare the kinematics of Bulge SRVs and Bulge Miras.

\section{References}

Bessel, M. S., Brett, J. M., Scholz, M., Wood, P. R., 1989, A\&A 213, 209

Blommaert J., 1992, thesis Leiden

Feast, M. W., Glass, I. A., Whitelock, P. A., Catchpole, R. M., 1989, MNRAS 241, 375

Kerschbaum, F., Hron, J., 1994, A\&A 106, 397 